\title{
Perceptions of competence and motor learning: performance criterion resulting in low success experience degrades learning
}

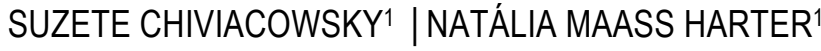

${ }^{1}$ Federal University of Pelotas - School of Physical Education

Correspondence to: Suzete Chiviacowsky, Federal University of Pelotas, School of Physical Education, Luís de Camões Street, 625, 96055-630 Pelotas, RS, Brazil Email: suzete@ufpel.edu.br

\begin{abstract}
AT A GLANCE
The present study investigated whether different opportunities to confirm good performance would impact learning. The findings reveal that learning is undermined when participants' perceptions of competence are reduced.
\end{abstract}

\begin{abstract}
BACKGROUND: Recent findings have provided converging evidence for the important role of perceptions of competence on motor learning.

AIM: In the present study we asked whether thwarting learners' need to feel competent by setting a relatively high criterion for "good" performance, thereby reducing their experience of success, would degrade learning.

METHOD: Participants practiced a coincident-anticipation timing task and received error feedback after every other trial (50\%) during the practice phase. One group (low success experience or LS) was informed before the beginning of practice that an error of 4 ms or less would be considered a good trial, whereas another group (high success experience or HS) was told that an error of $30 \mathrm{~ms}$ or less would be considered good performance. A third (control) group was not given a performance criterion.

RESULTS: During practice, participants in the LS and HS groups experienced good performance (i.e., were within their criterion range) on $6.3 \%$ and $57.8 \%$ of the feedback trials, respectively. On retention and transfer (non-dominant hand) tests without feedback one day after practice, absolute errors of the HS and control groups were significantly lower than those of the LS group. Participants in the HS group reported higher levels of self-efficacy than LS and control group participants. CONCLUSION: The results demonstrate that reducing learners' opportunities to experience success during practice degraded learning.

KEYWORDS: motor behavior | feedback | self-efficacy | timing
\end{abstract}

\section{INTRODUCTION}

Treated as a basic psychological need, competence is considered as essential for ongoing psychological growth and well-being. ${ }^{1,2}$ An individual's belief regarding his or her competence to complete a determined task is defined as perceived self-efficacy, which is considered to affect the quality of his cognitive, affective and decisional processes. ${ }^{3,4}$ Increased self-efficacy has been linked to improved performance in different domains and tasks. $^{5-7}$

Manipulating feedback, an important factor of practice, recent studies have been highlighting the importance of the learners' perceptions of competence in motor learning. For example, it has been observed that self-controlled learners prefer to receive feedback after more effective rather than less effective trials. ${ }^{8-10} \mathrm{~A}$ series of follow-up studies have demonstrated that feedback is indeed more effective for motor learning when provided after relatively good instead of bad trials. ${ }^{11-16}$

Benefits involving heightened perceptions of competence have also been observed when feedback suggested better-than-average performance. ${ }^{17-19}$ In these studies, participants receiving (false) positive social-comparative feedback in addition to veridical feedback were led to believe that their performance was better than average. These participants in turn demonstrated better learning compared to individuals receiving negative social-comparative feedback or control participants not provided with socialcomparative information. In another context, it was also observed that different conceptions of ability (or competence) could also have an effect on the learning of motor 
tasks. For instance, it has been demonstrated that information inducing malleable conceptions of ability, provided by feedback ${ }^{20}$ or instructions, ${ }^{21,22}$ resulted in more effective learning of motor tasks than information inducing fixed conceptions of ability.

Taken together, these findings highlight the important role that perception of competence may play in the motor learning process. Events that seem to support the perception of competence of learners tend to benefit motor learning, whereas events that seem to prevent the satisfaction of the need for competence tend to undermine motor learning. The objective of the present study was therefore to further examine the motivational impact of learners' perceptions of competence on the learning of motor skills. In a previous self-control study, it was observed that the opportunity to confirm good performance while choosing when to receive feedback is an important aspect of the benefits regularly observed for this specific kind of practice on motor learning. ${ }^{23}$ In this study, learners not able to confirm successful performance while asking for feedback information showed decreased learning. The present study has followed up on this finding by testing whether thwarting the need to feel competent by setting a relatively high criterion for "good" performance, thereby reducing their experience of success, would also degrade learning in contexts of practice where learners are not allowed to choose. Alongside competence, autonomy is considered to be a basic psychological need, ${ }^{24}$ with several studies demonstrating its impact on human behavior.25-27 Choices, or the exercise of control, have been shown to result in inherent rewards in different domains. ${ }^{27-31} \mathrm{In}$ fact, in autonomy supportive practice contexts involving self-controlled feedback, it has already been observed that learners provided with choices demonstrated not only higher learning, but also enhanced perceived competence ${ }^{32}$ compared to learners who are not allowed to choose, even when the latter received a feedback schedule mirroring their counterparts regarding trial accuracy. Thus, it is important to investigate whether different opportunities to confirm good performance would impact learning when dissociated from autonomy support effects.

If perceived competence is in fact a critical condition for motor learning, it would be expected that participants receiving a relatively high criterion for good performance, thereby decreasing their opportunities to experience success, would report lower levels of self-efficacy after practice and demonstrate reduced learning outcomes compared to participants receiving a relatively low criterion for good performance, thereby increasing their opportunities to experience success. Considering previous results within the literature demonstrating the effects of perceived competence in motor performance and learning, we hypothesized that participants receiving a high criterion for good performance, resulting in practice with low experience of success, would show decreased self-efficacy and degraded learning than participants for whom the criterion resulted in high experience of success or participants not receiving any performance criterion.

\section{METHODS}

\section{Participants}

Fifty-four university students (30 males, 24 females) with a mean age of 24.4 years (SD: 6.73) participated in the study. The participants had no prior experience regarding the experimental task and were not aware of the specific purpose of the study. 
The university's institutional review board approved the study, and participants gave their informed consent.

\section{Apparatus and task}

A Bassin anticipation timer (Model 35575, Lafayette Instruments, Lafayette, IN), an apparatus consisting of a $228-\mathrm{cm}$ long track with 48 light-emitting diodes (LEDs) on its surface, was used to measure temporal accuracy in anticipatory timing. The task consisted of pressing with the thumb of the preferred hand from a seated position while facing the apparatus, a hand-held switch coincident with the illumination of the (last) target light. A sequential illumination of the LEDs was created so the participants had the perception of a luminous red light moving down the runway; fifteen lights before the last one (target light) were obscured through a barrier placed on the top of the trackway in order to increase the difficulty of the task. A yellow warning light was used for all to cue the initiation of each trial. It was set to illuminate for a variable period of time (2-5 s), while the running light was set to apparently move at a constant speed of $20 \mathrm{mph}$. In order to measure temporal accuracy (absolute error, or $\mathrm{AE}$ ), the absolute difference between the target light illumination and the press of the switch was used.

\section{Procedure}

After completing the consent form, participants were randomly assigned to the three groups - High experience of success (HS), Low experience of success (LS) and Control - and introduced to the task. They were informed they should use the thumb of the preferred hand to press a hand-held switch coincidently with the illumination of the target light. They were also told that pressing the switch coincidently with the target light illumination would correspond to a 0 ms error. While participants in the control group did not receive any information regarding performance standard, participants in the HS group were told that an error of $30 \mathrm{~ms}$ or less would be considered good performance and participants in the LS group were informed that an error of $4 \mathrm{~ms}$ or less would constitute good performance. Feedback consisted of the number of milliseconds the switch was pressed before or after the illumination of the target light, including error direction (e.g., -27 $\mathrm{ms}$ ), and the groups were informed that they would receive feedback after every other practice trial $(50 \%)$. All participants performed 30 trials during the practice phase. Retention and transfer tests, performed 1 day later, consisted each of 10 trials without feedback, and were conducted in the same order for all participants. In the transfer test participants were asked to use their non-dominant hand to press the switch. It was performed five minutes after retention.

All participants were also asked to complete a self-efficacy questionnaire immediately before the beginning of practice, after the practice phase, and before the retention test. In this questionnaire, they were asked to rate their confidence level on a scale from 1 ("not at all") to 10 ("very") that their errors would be smaller than 50 and 30 ms after practice, the next day, or during retention, respectively. In addition, we were also interested in whether self-efficacy ratings would be able to predict learning, as observed in previous research. ${ }^{23,32-34}$

\section{Data Analysis}

AEs and VEs were averaged across blocks of five trials for the practice phase, and one block of ten trials for the retention and transfer tests. The practice data was analysed 
through analysis of variance (ANOVA), in a 3 (groups) $\times 6$ (blocks of five trials), with repeated measures on the last factor. For analysis of the retention and transfer test data, separate one-way ANOVAs were used. Self-efficacy ratings were averaged across the two task difficulty levels (50 and $30 \mathrm{~ms}$ ) and the subsequent analysis was conducted in a oneway ANOVA. In addition, a linear regression analysis was conducted in order to determine whether self-efficacy predicted performance on the retention and transfer tests. For significant results, partial eta-squared values $\left(\eta_{p}{ }^{2}\right)$ were used to indicate effect sizes. The alpha was set at .05 for all analyses.

\section{RESULTS}

\section{Temporal Accuracy}

Practice. Participants from all groups reduced their AEs (see Figure 1, left) during the practice phase. The main effect of block was significant, $F(5,255)=12.37, p<.01, \eta_{p}{ }^{2}$ $=.20$, while the main effect of group, $F(2,51)<1$, and the Group x Block interaction, $F(10$, $255)=1.27, p>.05$, were not significant. A similar pattern was observed for VEs (Figure 2, left) during this phase. The main effect of block was significant, $F(5,255)=11.31, p<.01$, $\eta_{p}{ }^{2}=.18$. The main effect of group, $F(2,51)<1$, and the Group $x$ Block interaction, $F(10$, $255)=1.16, p>.05$, were not significant. For the feedback trials, participants of the LS group had an error of $4 \mathrm{~ms}$ or less, indicating good performance, in $6.3 \%$ of the trials. Participants from the HS group in turn had an error of $30 \mathrm{~ms}$ or less in $57.8 \%$ of the feedback trials.

Retention. As can be observed in Figures 1 and 2 (middle), on the no-feedback retention test the LS group underperformed the other groups. The group main effect was significant for $\mathrm{AE}, F(2,51)=3.76, p<.05, \eta_{p}^{2}=.13$, and VE, $F(2,51)=5.37, p<.01, \eta_{p}{ }^{2}=.17$. Post-hoc tests confirmed that the HS group had smaller AEs and VEs than the LS group, $p s<.05$. There was no difference between the LS and the control group, $p s>.05$.

Transfer. During the no-feedback transfer test (see Figure 1, right), the LS group again underperformed the other groups regarding $A E$. The group main effect was significant, $F(2$, $51)=4.15, p<.05, \eta_{p}{ }^{2}=.14$. Similarly to retention, post-hoc tests confirmed that the HS group had smaller AEs than the LS group, $p<.05$, but did not differ from the control group, $p>.05$. No differences between groups (Figure 2, right) were found for $\operatorname{VE}, F(2,51)=$ $1.51, p>.05$. 


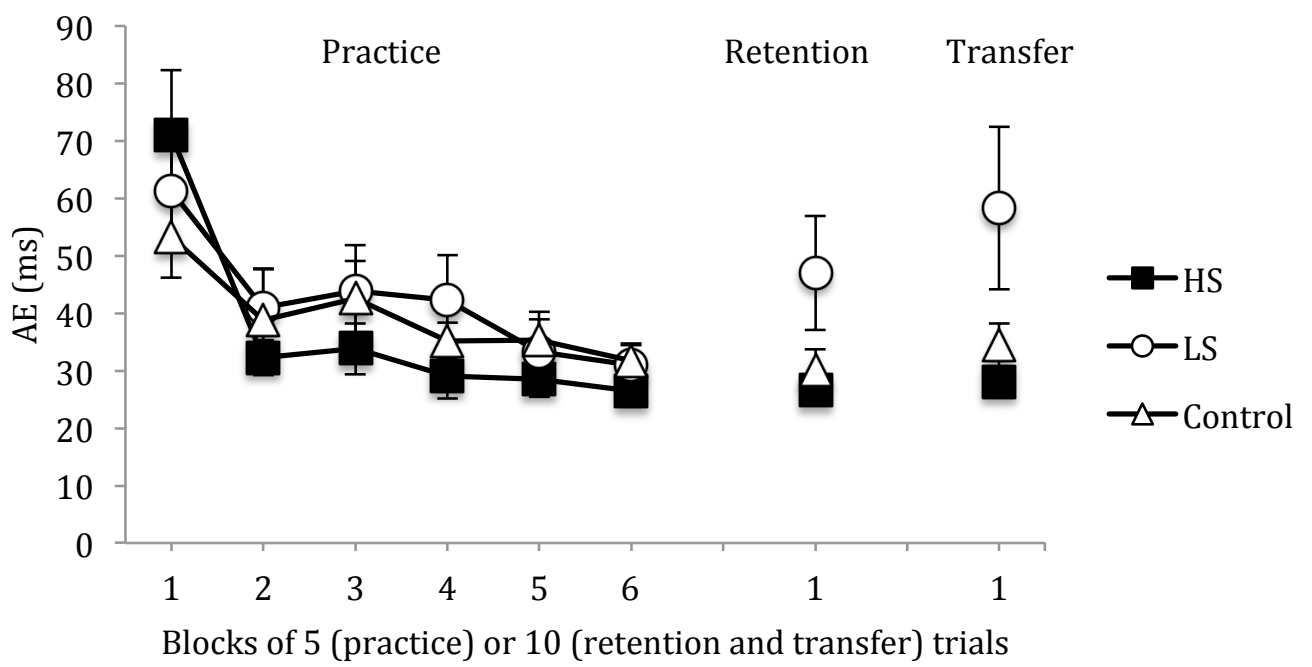

Figure 1. Absolute errors for the groups during practice (Day 1) and delayed retention and transfer tests (Day 2). Note: Error bars indicate standard errors.

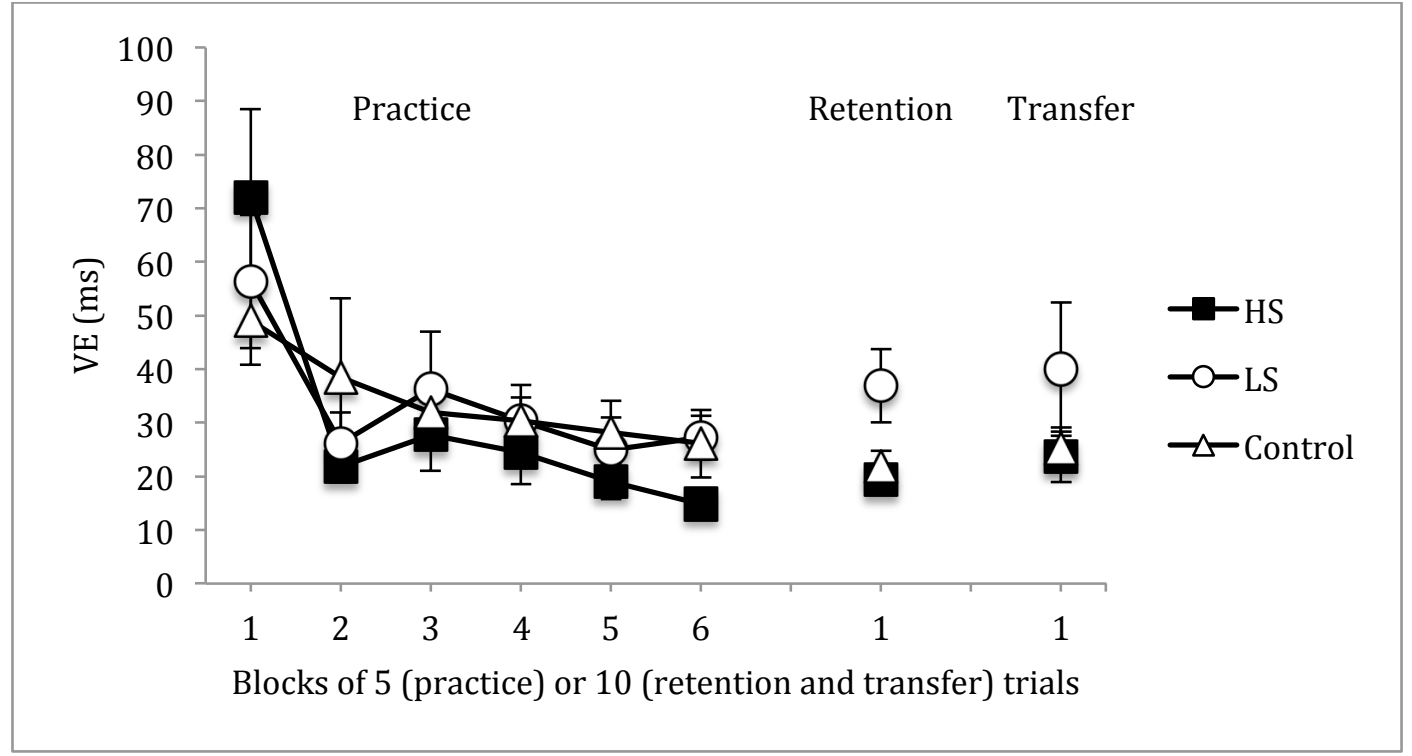

Figure 2. Variable errors for the groups during practice (Day 1) and delayed retention and transfer tests (Day 2). Note: Error bars indicate standard errors. 


\section{Self-efficacy}

Before practice, there was no evident difference between the groups regarding self-efficacy levels (see Figure 3). The main effect of group, $F(2,51)<1$, was not significant.

After practice, the main effect of group, $F(2,51)=4.24, p<.05, \eta_{p}^{2}=.14$, was significant. Post-hoc tests confirmed that the HS group reported higher significant SE levels than the LS group, $p<.05$, and higher marginally significant SE levels, $p=.064$, than the control group.

A difference between groups was also observed on the second day before the retention test. The main effect of group, $F(2,51)=4.77, p<.05, \eta_{p}^{2}=.16$, was significant. Post-hoc tests showed higher levels of self-efficacy for the HS group compared to LS and control groups, ps $<.05$.

In order to determine whether self-efficacy could be considered to be a significant predictor of learning after the practice phase and before the retention test, linear regressions analyses were conducted. The results show that self-efficacy measured immediately after practice significantly predicted the retention, $F(2,53)=3.31, p<.05, \mathrm{R}$ $=.08$, and transfer, $F(2,53)=3.23, p<.05, R=.07$, tests performances, explaining $33.9 \%$ and $33.5 \%$ of the variances, respectively.

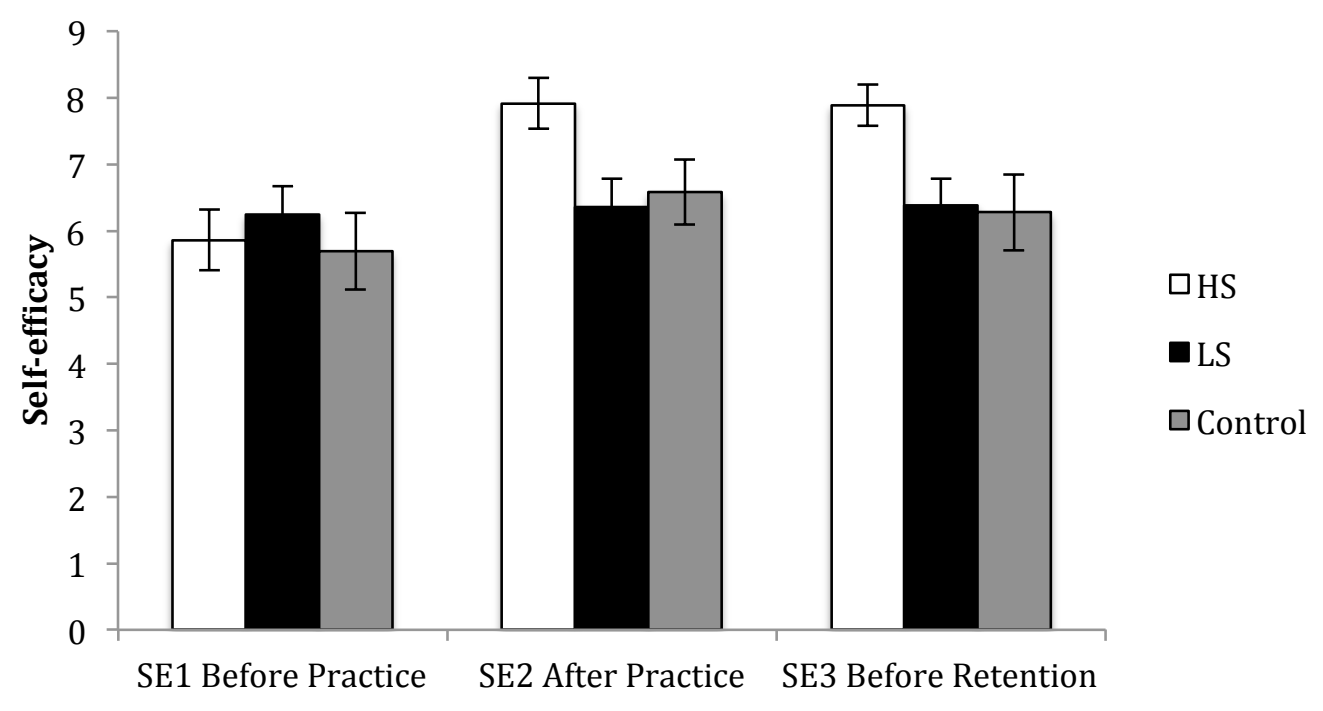

Figure 3. Self-efficacy scores before and after practice (Day 1), and before retention (Day 2). Note: Error bars indicate standard errors.

\section{DISCUSSION}

In the present study we tested whether preventing learners' need to feel competent by setting a relatively high criterion for "good" performance, thereby reducing their experience of success, would degrade motor learning. As stated by the selfdetermination theory, ${ }^{1,2}$ competence influences intrinsic motivation through the satisfaction of a basic psychological need; the fulfilment of the competence need is considered to predict improved functioning and learning, while thwarting the competence need has been 
associated with lower functioning as well as with symptoms of ill-being in different domains. $^{1}$

The present findings reveal that learning is undermined when participants' experience of success is reduced as indicated by the performance during the retention and transfer tests. Participants from the low experience of success group showed decreased learning compared with participants in the high experience of success and control groups. The findings indicate that individuals are sensitive to their experience of success during practice, responding differently under high or low criterions of good performance. The results are in agreement with previous findings showing improved learning when learners receive feedback after more efficient trials rather than less efficient ones, ${ }^{11-15}$ when feedback suggests positive instead of negative social-comparison, ${ }^{17-19,34}$ or when feedback induces a malleable conception of ability, a condition where learners errors are not viewed as a threat to the self 20 compared with a fixed conception of ability.

Furthermore, participants for whom the experience of success was high exhibited greater self-efficacy compared to participants with low levels of success experience and controls. Self-efficacy, in addition, predicted learning. So, the use of a relatively low criterion for good performance, in turn creating a higher experience of success in the task, was more motivational for learners. Previous studies have showed that enhancing expectations of performance may indeed influence learners' perceptions of competence. ${ }^{7}$, $15,34,35$ In line with the present findings, self-efficacy has also been showing to predict motor learning in other practice contexts. ${ }^{23,} 32,33$ Self-efficacy beliefs have in fact long been considered to affect the human functioning through motivational, affective and decisional processes, influencing how people choose challenges to confront, how much effort to expend or how long to persist, and whether failures are motivating or demoralizing. ${ }^{3-5,36}$ Self -efficacy has also demonstrated to be both, cause and effect of motor performance. ${ }^{37}$

Underlying mechanisms explaining how the experience of successful performance affects motor learning have been suggested. For example, individuals facing more doubt regarding their own competence, like the situation faced by participants of the low success group, can tend to turn their attention inwardly, occupying themselves with evaluative concerns. ${ }^{38}$, 39 These self-directed thoughts, probably caused by anxiety, can be considered detrimental to motor learning, interfering with task-focusing thinking.40-42 Caused by worries about task performance, a focus on the self can lead individuals to selfregulatory processes, resulting in "micro-choking" episodes with attempts to control thoughts and emotions, degrading learning. ${ }^{42}$

The present results are important for theoretical and practical reasons. They give further support to the importance of perceptions of competence for motor learning, showing that learning can be undermined when participants' experience of success is reduced. More specifically, they demonstrate that the effects on motor learning in terms of setting relatively low or high criterion for good performance, respectively increasing or reducing learner's experience of success, are mediated by perceived competence. The findings have implications for practical situations where motivation plays an important role, for example educational and sporting settings. Instructors or coaches could make use of strategies, such as establishing suitable criterions for good performance in order to increase learner's perceptions of competence during practice. The development of situations where learners are able to more frequently experience good performance may have the potential to further increase interest and persistence in the long run, as well as learning. On the other hand, practice conditions where very difficult criterions for good 
performance are being set should be avoided since they can reduce learners' perceptions of competence, therefore undermining learning.

The present findings have been obtained with young adults learning a simple laboratory timing task. Future research could examine whether increasing learners' experience of success, by setting a relatively easy criterion for good performance, would enhance perceptions of competence and learning in different population, for example children, older adults, or individuals with motor or intellectual disabilities. It would also be valuable if future studies could replicate the present findings in contexts with higher ecological validity, for example sports tasks. Another aspect for research could be the identification of other variables that could potentially be affected by practices with different criterions of good performance, for example the affective dimensions. Such studies could establish further supportive evidence regarding the importance of perceptions of competence for motor learning.

\section{REFERENCES}

1. Deci EL, Ryan RM. The "what" and "why" of goal pursuits: Human needs and the selfdetermination of behavior. Psychol Inq 2000; 11: 227-68.

2. Deci EL, Ryan RM. Self-Determination Theory: A Macrotheory of Human Motivation, Development, and Health. Can Psychol 2008; 49: 182-5.

3. Bandura A. Self-efficacy: toward a unifying theory of behavioral change. Psychol Rev 1977; 84: 191-215.

4. Bandura A. On the Functional Properties of Perceived Self-Efficacy Revisited. J Manag 2012; 38: 9-44.

5. Bandura A. Perceived self-efficacy in cognitive-development and functioning. Educ Psychol 1993; 28: 117-48.

6. Feltz $\mathrm{DL}$, Chow GM, Hepler TJ. Path analysis of self-efficacy and diving performance revisited. J Sport Exerc Psychol 2008; 30: 401-11.

7. Hutchinson JC, Sherman T, Martinovic N, Tenenbaum G. The Effect of Manipulated SelfEfficacy on Perceived and Sustained Effort. J Appl Sport Psychol 2008; 20: 457-72.

8. Chiviacowsky $S$, Wulf $\mathrm{G}$. Self-controlled feedback: does it enhance learning because performers get feedback when they need it? Res Q Exerc Sport 2002; 73: 408-15.

9. Patterson JT, Carter M. Learner regulated knowledge of results during the acquisition of multiple timing goals. Hum Movement Sci 2010; 29: 214-27.

10. Fairbrother JT, Laughlin DD, Nguyen TV. Self-controlled feedback facilitates motor learning in both high and low activity individuals. Front Psychol 2012; 3.

11. Badami R, VaezMousavi M, Wulf G, Namazizadeh M. Feedback After Good Versus Poor Trials Affects Intrinsic Motivation. Res Q Exerc Sport 2011; 82: 360-4.

12. Badami R, VaezMousavi M, Wu G, Namaziza M. Feedback About More Accurate Versus Less Accurate Trials: Differential Effects on Self-Confidence and Activation. Res Q Exerc Sport 2012; 83: 196-203. 
13. Chiviacowsky S, Wulf G. Feedback after good trials enhances learning. Res Q Exerc Sport 2007; 78: 40-7.

14. Chiviacowsky S, Wulf G, Wally R, Borges T. Knowledge of Results After Good Trials Enhances Learning in Older Adults. Res Q Exerc Sport 2009; 80: 663-8.

15. Saemi E, Porter JM, Ghotbi-Varzaneh A, Zarghami M, Maleki F. Knowledge of results after relatively good trials enhances self-efficacy and motor learning. Psychol Sport Exerc 2012; 13: $378-82$.

16. Saemi E, Wulf G, Varzaneh AG, Zarghami M. Feedback after good versus poor trials enhances motor learning in children. Rev Bras Educ Fís Esporte 2011; 25: 673-81.

17. Avila LTG, Chiviacowsky S, Wulf G, Lewthwaite R. Positive social-comparative feedback enhances motor learning in children. Psychol Sport Exerc 2012; 13: 849-53.

18. Lewthwaite R, Wulf $G$. Social-comparative feedback affects motor skill learning. Q J Exp Psychol 2010; 63: 738-49.

19. Wulf G, Chiviacowsky S, Lewthwaite R. Normative Feedback Effects on Learning a Timing Task. Res Q Exerc Sport 2010; 81: 425-31.

20. Chiviacowsky S, Drews R. Effects of generic versus non-generic feedback on motor learning in children. PLoS One 2014; 9: e88989.

21. Drews R, Chiviacowsky S, Wulf $\mathrm{G}$. Children's motor skill learning is influenced by their conceptions of ability. JMLD 2013; 1: 38-44.

22. Wulf G, Lewthwaite R. Conceptions of ability affect motor learning. J Mot Behav 2009; 41: 461-7.

23. Chiviacowsky S, Wulf $G$, Lewthwaite R. Self-controlled learning: the importance of protecting perceptions of competence. Front Psychol 2012; 3.

24. Deci EL, Ryan RM. Self-determination theory: A macrotheory of human motivation, development, and health. Can Psychol 2008; 49: 182.

25. Cordova DI, Lepper MR. Intrinsic motivation and the process of learning: Beneficial effects of contextualization, personalization, and choice. J Educ Psychol 1996; 88: 715.

26. Hackman JR, Oldham GR. Motivation through the design of work: Test of a theory. Organ Behav Hum Perform 1976; 16: 250-79.

27. Tafarodi RW, Milne AB, Smith AJ. The confidence of choice: Evidence for an augmentation effect on self-perceived performance. Pers Soc Psychol B 1999; 25: 1405-16.

28. Catania AC. Freedom and knowledge: An experimental analysis of preference in pigeons. J Exp Anal Behav 1975; 24: 89-106.

29. Catania AC, Sagvolden T. Preference for free choice over forced choice in pigeons. J Exp Anal Behav 1980; 34: 77-86.

30. Leotti LA, Delgado MR. The inherent reward of choice. Psychol Sci 2011.

31. Tiger JH, Hanley GP, Hernandez E. An evaluation of the value of choice with preschool children. J Appl Behav Anal 2006; 39: 1.

32. Chiviacowsky S. Self-controlled practice: Autonomy protects perceptions of competence and enhances motor learning. Psychol Sport Exerc 2014; 15: 505-10. 
33. Stevens D, Anderson DI, O'Dwyer NJ, Williams AM. Does self-efficacy mediate transfer effects in the learning of easy and difficult motor skills? Conscious Cogn 2012; 21: 1122-8.

34. Wulf G, Chiviacowsky S, Cardozo PL. Additive benefits of autonomy support and enhanced expectancies for motor learning. Hum Mov Sci 2014; 37: 12-20.

35. Chauvel G, Wulf $G$, Maquestiaux $F$. Visual illusions can facilitate sport skill learning. Psychon Bull Rev 2015; 22: 717-21.

36. Bandura A. Social cognitive theory: An agentic perspective. Annu Rev Psychol 2001; 52: $1-26$.

37. Moritz SE, Feltz DL, Fahrbach KR, Mack DE. The relation of self-efficacy measures to sport performance: A meta-analytic review. Res Q Exerc Sport 2000; 71: 280-94.

38. Bandura A. Self-efficacy mechanism in human agency. Am Psychol 1982; 37: 122.

39. Bandura A, Wood R. Effect of perceived controllability and performance standards on selfregulation of complex decision making. J Pers Soc Psychol 1989; 56: 805.

40. Sarason IG. Stress, anxiety, and cognitive interference: reactions to tests. J Pers Soc Psychol 1984; 46: 929.

41. Wine J. Test anxiety and direction of attention. Psychol Bull 1971; 76: 92.

42. Wulf $\mathrm{G}$, Lewthwaite R. Effortless motor learning? An external focus of attention enhances movement effectiveness and efficiency. In: Bruya B, editor. Effortless attention: A new perspective in attention and action. Cambridge, MA: MIT Press; 2010. p. 75-101.

Citation: Chiviacowsky S, Harter NM. Perceptions of competence and motor learning: performance criterion resulting in low success experience degrades learning. BJMB. 2015: 9(1): 1-10.

Editor: Joao A. C. Barros, California State University Fullerton, Fullerton, CA, USA.

Copyright: @ 2015 Chiviacowsky S and BJMB. This is an open-access article distributed under the terms of the

Creative Commons Attribution-NonCommercial-NoDerivatives 4.0 International License which permits unrestricted use, distribution, and reproduction in any medium, provided the original author and source are credited.

Competing interests: The authors have declared that no competing interests exist.

Download: http://socibracom.com/bjmb/index.php/bjmb/issue/view/24 